\title{
Dexmedetomidine attenuates cerebral ischemia-reperfusion injury in rats by inhibiting the JNK pathway
}

\author{
Bingxiao Zhao, Da Li, Shuchi Zhang, Long He, Yanqiu Ai \\ Department of Anesthesiology, the First Affiliated Hospital of Zhengzhou University, Zhengzhou, China \\ Contributions: (I) Conception and design: Y Ai; (II) Administrative support: Y Ai; (III) Provision of study materials or patients: B Zhao; (IV) Collection \\ and assembly of data: All authors; (V) Data analysis and interpretation: B Zhao; (VI) Manuscript writing: All authors; (VII) Final approval of \\ manuscript: All authors. \\ Correspondence to: Yanqiu Ai. Department of Anesthesiology, The First Affiliated Hospital of Zhengzhou University, No.1, Jianshe Road, Zhengzhou \\ 450000, China. Email: Aiyanqiu182@163.com.
}

Background Cerebral ischemic reperfusion injury (CI/RI) is a common cerebrovascular disease with high morbidity and disability that threatens human health. This study was conducted to explore the effects of dexmedetomidine (Dex) on the c-Jun N-terminal kinase (JNK) pathway in CI/RI, and to provide a theoretical basis for the recovery of brain function after cerebral ischemia.

Methods: Sprague Dawley $(\mathrm{SD})$ rats $(\mathrm{n}=24)$ were randomly divided into Sham, Sham + Dex, Sham + yohimbine (Yoh) + Dex, Sham + SP600125, ischemic reperfusion (I/R), I/R + Dex, I/R + Yoh + Dex, and I/R + SP600125 groups, and a focal cerebral ischemia reperfusion rat model was established by linear thrombus. The neurological deficit score and infarct volume were measured. Wet/dry weight ratios were used to measure brain water content, and cerebral infarct volume was determined by 2, 3, 5-triphenyltetrazolium chloride (TTC) staining. The cellular distribution of p-JNK and cleaved caspase-3 were examined using immunofluorescent staining (IF) and the total JNK and p-JNK were determined by Western blotting (WB).

Results: Compared with the Sham group, the rats in I/R, I/R + Dex, I/R + Yoh + Dex, and I/R + SP600125 groups developed hemiparesis of the left forelimb at different levels with a higher neurological deficit score, brain water content, infarct volume, and markedly upregulated expression of cleaved caspase-3, p-JNK $(\mathrm{P}<0.05)$. Compared with the I/R group, the neurological deficit score, brain water content, infarct volume, and expression of cleaved caspase-3, p-JNK were markedly decreased in I/R + Dex, I/R + Yoh + Dex, and $\mathrm{I} / \mathrm{R}+\mathrm{SP} 600125$ groups $(\mathrm{P}<0.05)$, and compared with the $\mathrm{I} / \mathrm{R}+\mathrm{Dex}$ group, the neurological deficit score, brain water content, infarct volume, and expression of cleaved caspase-3, p-JNK were markedly increased in the $\mathrm{I} / \mathrm{R}+$ Yoh + Dex group $(\mathrm{P}<0.05)$. Double immunofluorescence staining showed there was a strong colocalization between $\mathrm{p}-\mathrm{JNK}$ and the astroglial marker GFAP.

Conclusions: The JNK signaling pathway is involved in CI/RI. Inhibition of the JNK pathway blocked caspase-3 activation which can decrease CI/RI. Dex can alleviate cerebral CI/RI in rats by increasing $\alpha 2-$ adrenergic receptor and blocking JNK phosphorylation and activation of caspase-3.

Keywords: Dexmedetomidine (Dex); cerebral ischemic reperfusion injury (CI/RI); Jun N-terminal kinase (JNK)

Submitted Apr 20, 2021. Accepted for publication Jun 08, 2021.

doi: $10.21037 /$ apm-21-1218

View this article at: https://dx.doi.org/10.21037/apm-21-1218 


\section{Introduction}

Cerebrovascular disease is a serious threat to human health with a high incidence, high disability rate, and high recurrence rate, among which $60-80 \%$ are ischemic in nature (1). Cerebral ischemic reperfusion injury (CI/RI) occurs when ischemic tissue and organs cannot recover their function and structure after blood reperfusion, which can also aggravate an initial injury. Mitogen activated protein kinase (MAPK) is a highly conserved signal transduction mechanism including the c-Jun $\mathrm{N}$-terminal kinase (JNK) pathway, P38 MAPK pathway, and extracellular signalregulated kinase (ERK) pathway. The JNK family is a member of the MAPK superfamily, which plays a central role in signal transduction. The JNK-mediated signaling pathway is involved in a variety of processes including building cytoskeleton, shape maintenance, cell proliferation, cell differentiation, cell pathological process, cell apoptosis, and cell malignant transformation (2). Dexmedetomidine (Dex) is a highly selective $\alpha 2$ adrenergic receptor agonist. It has the effects of inhibiting sympathetic nerves, sedation, hypnosis, analgesia, reducing anesthesia, and reducing postoperative delirium. It is widely used in surgery and ICU sedation. Dex was approved by the US Food and Drug Administration (FDA) for patients requiring intensive care with mechanical ventilation (3) and exerts its function by interacting with $\alpha 2$-adrenergic receptors. Dex is widely used clinically because it is easy to arouse and respiratory depression characteristics. Yohimbine (Yoh) is a selective $\alpha 2$ adrenergic receptor blocker, which can expand vascular smooth muscle, increase peripheral parasympathetic tension, and reduce sympathetic tension. It is widely used in the research of nervous, cardiovascular and endocrine systems.

Recent studies have shown that Dex exerts its antiapoptotic effects via MAPK signaling pathways and can inhibit cell apoptosis by the activation of extracellular signal-regulated kinase 1 and 2 (ERK1/2) phosphorylation in vivo and in vitro (4). In epithelial cell apoptosis models induced by high bilirubin concentration, Dex can improve the survival of cells by inhibiting caspase-3 activation (5) and can reduce isoflurane-induced apoptosis of hippocampal neurons in neonatal rats by blocking the activation of $\mathrm{p} 38$ and JNK (6). Thus, we hypothesize that Dex attenuates CI/ RI by inhibiting the JNK pathway.

In this study, we sought to establish a model of CI/RI in rats to explore whether the role of Dex in CI/RI is related to the JNK pathway given by Dex, Yoh, $\alpha 2$-adrenergic receptor antagonists, and SP600125, a generic inhibitor of JNK kinase These results may provide a rationale for therapeutic strategies to recover CI/RI patients in the clinic. We present the following article in accordance with the ARRIVE reporting checklist (available at https://dx.doi. org/10.21037/apm-21-1218).

\section{Methods}

\section{Experimental animals}

A total of 196 SD rats with an average body weight of 250-280 g were purchased from the Henan Experimental Animal Center. All animal experiments complied with the relevant ethical regulations. A protocol was prepared before the study without registration. Experiments were performed under a project license [NO.: SCXK (Yu) 20150004] granted by the ethics committee of the First Affiliated Hospital of Zhengzhou University, in compliance with Chinese national guidelines for the care and use of animals.

\section{Grouping and modeling of experimental animals}

\section{Grouping of experimental animals}

SD rats were randomly divided into Sham, Sham + Dex, Sham + Yoh + Dex, Sham + SP600125, I/R, I/R + Dex, I/ $\mathrm{R}+$ Yoh + Dex, and I/R + SP600125 groups. In the nonsham groups, the common carotid artery (CCA), external carotid artery (ECA), and internal carotid artery (ICA) were separated, and vascular ligation and thread insertion was performed. A CI/RI model was established in these groups with ischemia. Rats in the Sham + Yoh + Dex and I/R + Yoh + Dex group were intraperitoneally injected with $0.5 \mathrm{mg} / \mathrm{kg}$ Yoh 30 min before vessel separation or ischemia, and Dex was pumped $3 \mu \mathrm{g} / \mathrm{kg}$ immediately after vessel separation or ischemia by a tail vein pump. The pump was completed within $5 \mathrm{~min}$, followed by $6 \mu \mathrm{g} \cdot \mathrm{kg}^{-1} \cdot \mathrm{h}^{-1}$ continuous pumping for $2 \mathrm{~h}$. The Sham + Dex and the I/R + DEX group were given the same amount of normal saline $30 \mathrm{~min}$ before vessel separation or ischemia, and the Sham and I/R group were given the same amount of normal saline. For the Sham + SP600125 and I/R + SP600125 groups, $10 \mu \mathrm{L}$ was injected into the lateral ventricle $30 \mathrm{~min}$ before blood vessel separation or modeling, with SP600125 dissolved in 1\% dimethyl sulfoxide, DMSO at a concentration of $30 \mathrm{mg} / \mathrm{mL}$.

\section{Lateral ventricle injection}

Rats were first fasted for $12 \mathrm{~h}$ and drank water freely, then 
SP600125 was injected into the lateral ventricle $30 \mathrm{~min}$ before modeling. SP600125 $10 \mu \mathrm{L}$ was then extracted with a microsampler which was fixed on the microsampler clamp. The anesthetic method was to inject $10 \%$ chloral hydrate $300 \mathrm{mg} / \mathrm{kg}$ intraperitoneally and fix it on the stereotaxer of the rat brain when the righting reflex disappeared. The hair on the top of the rat was cut off, and the skin on the top of the head was disinfected. A $1 \mathrm{~cm}$-long longitudinal incision was then made along the sagittal suture in the middle of the scalp to expose the skull and anterior fontanelle. The fontanelle was defined as the coordinate, and the right $1.5 \mathrm{~mm}$ and the back $1.1 \mathrm{~mm}$ were selected to the injection puncture points of the lateral ventricle. After the microsyringe was directly inserted into the needle $4.5 \mathrm{~mm}$, $10 \mu \mathrm{L}$ SP600125 was slowly injected into the lateral ventricle. The microsyringe was withdrawn outward every $2 \mathrm{~min}$ to $1 \mathrm{~mm}$ to prevent rapid outflow of CSF after $1 \mathrm{~min}$. When all the microsyringes were removed, the skin was immediately sutured to disinfect.

\section{Establishment of the RI/CI model}

The Middle Cerebral Artery Occlusion (MCAO) model was established using the suture-occluded method (7) and all surgeries were performed after rats were weighed. Rats were intraperitoneally injected with anesthesia until loss of the righting reflex, then placed in a supine position with body temperature maintained at $37 \pm 0.5^{\circ} \mathrm{C}$ throughout the experiment. The incision was sutured and disinfected with iodophor, and tissues carefully dissociated, without injury to the vagus nerve. The right CCA, ECA, and ICA were then carefully exposed. The ECA and proximal end of the CCA were ligated with one silk thread, and another was passed through the bottom of the proximal bifurcation of the ICA, which was knotted but not tightened for reserve. The ICA was then closed with an arterial clip temporarily near the intracranial side of the spare suture. A small incision was made at the proximal end of the CCA bifurcation, and a thread plug was inserted through the incision. When the thread was formed, the thread knot was gently tightened, the artery clip was loosened, and the thread plug was gently sent into the intracranial space. When slight resistance was felt and the depth was about $17-19 \mathrm{~mm}$, the head tip was considered to have reached the beginning of the middle cerebral artery (MCA) to completely block arterial blood supply. The appearance of a lighter iris color and Horner's sign indicated success of the model preparation. The incision was sutured layer by layer, leaving the end of the thread plug outside the incision. After 90 minutes of ischemia, the thread plug was gently withdrawn to the point of resistance, and its head end was considered to have returned to the bifurcation of the CCA. At this time, the blood supply of the MCA was restored, and reperfusion injury was established as rat brain ischemia reperfusion (IR). Excess thread was then cut off. The rats in Sham, Sham + Dex, Sham + Yoh + Dex, and Sham + SP600125 groups received the same surgery as the ischemia group without ligation or ischemia. Dex, Yoh, and SP600125 were administered according to the experimental design, and the other operations were the same as those in the ischemia group. Body temperature was maintained around $37^{\circ} \mathrm{C}$, and all rats had free access to food and water. Vital signs were closely observed, and the respiratory tract was kept unobstructed.

\section{Evaluation criteria}

\section{Neurological function scoring}

Neurological evaluations were performed 24 hours after reperfusion by using a neurological deficit scoring scale adapted from the Longa 5-grade scale, and analyses were performed by a single investigator who was blinded to grouping. The scale was carried out as follows: 0 point: no symptoms of neurological deficit; 1 point: the contralateral forelimb of the lesion could not be completely straightened when the tail was lifted; 2 points: turning in a circle to the paralyzed side when walking; 3 points: falling to the opposite side of the lesion while walking; 4 points: failing to walk spontaneously and loss of consciousness.

\section{Exclusion criteria}

Rats were excluded if any of the following were present: (I) no symptoms of nerve injury; (II) subarachnoid hemorrhage; (III) no ischemic pathological changes were observed under microscope; (IV) rats died before the observation time.

\section{Tissue samples and methods}

\section{Cerebral infarction area}

2, 3, 5-triphenyltetrazolium chloride (TTC) is a fat-soluble photosensitive complex. The reaction between TTC and succinate dehydrogenase in normal tissues turns red, whereas it turns pale in ischemic tissues due to the decrease in dehydrogenase activity.

The brain infarct area was determined from six random MCAO rats in each group. Rats were deeply anesthetized and their brains were removed with ice in the following 
manner: an incision was made along the skull, and the skin and fascia were cleared. The occipital bone was then removed using a micro rongeur to expose the brainstem, cerebellum, and posterior margin of the parietal bone, and the forceps lifted the posterior edge of the parietal bone sideways to expose the cerebral hemispheres. Bone forceps were used to remove the temporal bone on both sides and part of the frontal bone to fully expose the brain tissue. The dura mater and the connecting nerve were then cut to remove the entire brain from the base of the skull. The whole procedure was performed on ice to avoid liquefaction of brain tissue. Frozen brains were cryosectioned by microtome at $-20{ }^{\circ} \mathrm{C}$ for $20-25 \mathrm{~min}$, and after fixation, the cerebrum was sectioned coronally and the cerebellum and brainstem were removed. The positions were placed at the anterior pole and the midpoint of the optic chiasma, the funnel stalk, and the caudal pole of the posterior lobe, with five slices cut once at about $2 \mathrm{~mm}$. Brain slices were incubated in a $2 \%$ TTC solution at $37{ }^{\circ} \mathrm{C}$ for $10 \mathrm{~min}$, during which time they were intermittently gently shaken to make them uniformly colored. After staining, the slices were removed and fixed in 4\% paraformaldehyde, and the infarct volume was measured using the ImageJ program and the following formula:

$$
\frac{\text { infarct area } \times(1-(\text { the ipsilateral area }- \text { the contralateral area }) / \text { the contralateral area })}{\text { Whole brain volume }} \times 100 \%
$$

\section{Brain water content measurement}

Six rats in each group were randomly selected to measure the water content of brain tissue, $24 \mathrm{~h}$ after MCAO. Ischemic lateral brain tissue was taken and immediately weighed to obtain the wet weight (WW), and after drying to a constant weight, the dry weights (DW) were obtained. The brain water content was measured as the following formula:

$$
\text { brain water content }=\frac{W W-D W}{W W} \times 100 \%
$$

\section{IF on 7 NK and cleaved caspase-3 levels}

Six rats in each group were randomly selected for IF detection $24 \mathrm{~h}$ after MCAO, and the fixed tissue samples were dehydrated, paraffin-embedded, and sectioned. The membranes were then incubated with antibodies (JNK at $1: 1,000$, cleaved caspase 3 at $1: 200$ ) overnight at $4^{\circ} \mathrm{C}$, and finally with goat anti-rabbit antibodies (1:200). After the sections were sealed, photos were taken and analyzed by fluorescence microscopy.

\section{WB on p- $7 N K$ and cleaved caspase-3 levels}

A number of $200 \mathrm{mg}$ right upper frontal parietal cortex pieces of rats were placed into the ball of the precooling homogenizer, immediately supplemented with 1,000 iL of lysate, and grinded on ice for $5 \mathrm{~min}$, three times. The mixture was then centrifuged at $3,000 \times \mathrm{g}$ for $15 \mathrm{~min}$ at $4{ }^{\circ} \mathrm{C}$, the supernatant collected, and the protein concentration calculated with a BCA protein assay kit. Subsequently, the membranes were blocked at room temperature for $2 \mathrm{~h}$ in fat-free milk. The membranes were then incubated with primary antibody against JNK (1:1,000 dilution) and p-JNK (1:500 dilution) overnight at $4{ }^{\circ} \mathrm{C}$ and goat anti-rabbitHRP IgG (1:10,000 dilutions) for $2 \mathrm{~h}$ at room temperature. Finally, ECL solution A and B were mixed 1:1 and the protein bands were visualized and analyzed using the ProteinSimple chemiluminescence system. The exposure time was adjusted according to the signal intensities, and photos were saved for further study.

\section{Statistical analysis}

All data were statistically processed by SPSS 17.0. The measurement data were expressed as mean $\pm \operatorname{standard}(\bar{x} \pm s)$ deviation, and one-way analysis of variance was applied for comparisons among multiple groups. If the differences were significant, the Least-significant Difference (LSD)-t method was used for a pairwise comparison. The test of significance was taken as $\mathrm{P}$ value $<0.05$.

\section{Results}

\section{Neurological function scoring}

Rats in the Sham, Sham + Dex, Sham + yohimbine $($ Yoh $)+$ Dex, and Sham + SP600125 groups showed no neurological impairment symptoms from waking up to the point before brain tissue extraction $(\mathrm{P}>0.05)$. Compared with the Sham group, rats in the I/R, I/R + Dex, I/R + Yoh + Dex, and I/R + SP600125 groups all had hemiplegia of the left forelimb with differing degrees and significantly increased neurological function scores 
Table 1 Comparison of neurological function scores in each group $(\bar{x} \pm \mathrm{s}, \mathrm{n}=24)$

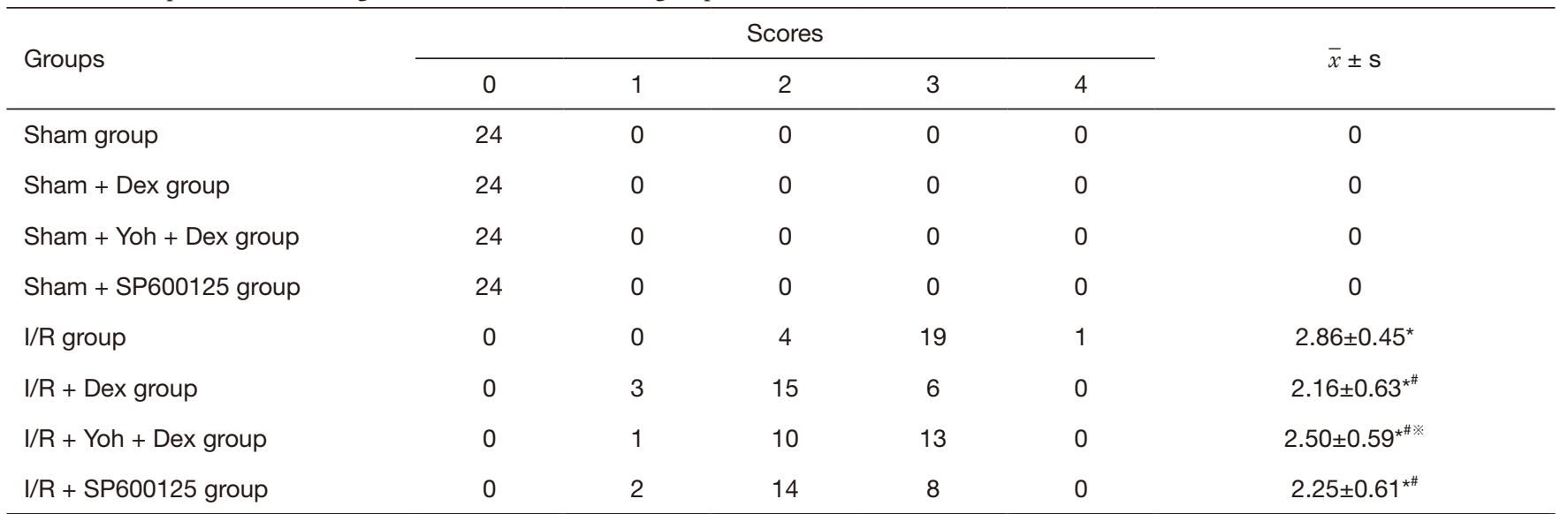

* compared with the Sham group, $\mathrm{P}<0.05$; " compared with the $\mathrm{I} / \mathrm{R}$ group, $\mathrm{P}<0.05$; ${ }^{*}$ compared with the $\mathrm{I} / \mathrm{R}+\mathrm{Dex}$ group, $\mathrm{P}<0.05$. Dex, dexmedetomidine; Yoh, yohimbine; I/R, ischemic reperfusion.

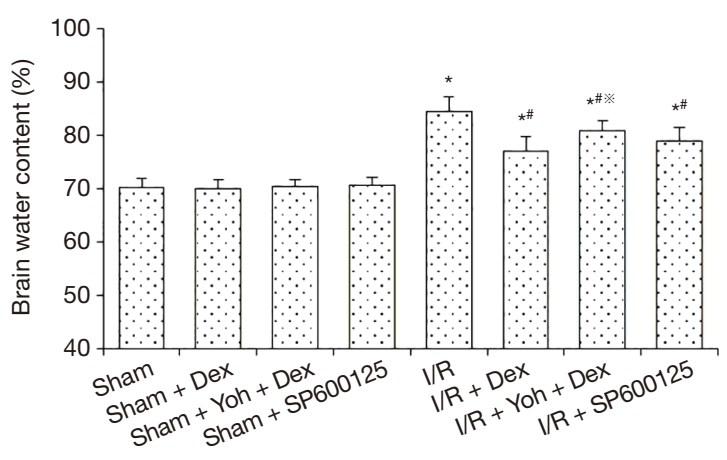

Figure 1 Comparison of brain water content in each group $(\mathrm{n}=6)$. * compared with the Sham group, $\mathrm{P}<0.05$; " compared with the I/R group, $\mathrm{P}<0.05$; * compared with the $\mathrm{I} / \mathrm{R}+\mathrm{Dex}$ group, $\mathrm{P}<0.05$. Dex, dexmedetomidine; Yoh, yohimbine; I/R, ischemic reperfusion.

$(\mathrm{P}<0.05)$. Compared with the $\mathrm{I} / \mathrm{R}$ group, the neurological function scores in the I/R + Dex, I/R + Yoh + Dex, and I/R + SP600125 groups were significantly decreased $(\mathrm{P}<0.05)$, and the neurological function scores in $\mathrm{I} / \mathrm{R}+$ Yoh + Dex group were significantly higher than those in the I/R + Dex group $(\mathrm{P}<0.05)$ (see Table 1).

\section{Brain water content measurement}

There was no statistical difference in brain water content measurement among the Sham, Sham + Dex, Sham + Yoh + Dex, and Sham + SP600125 groups, $24 \mathrm{~h}$ after surgery ( $P>0.05)$. Compared with the Sham group, the rats in $I / R$, $\mathrm{I} / \mathrm{R}+$ Dex, I/R + Yoh + Dex, and I/R + SP600125 groups had lower brain water content $(\mathrm{P}<0.05)$ and compared with the I/R + Dex group, the brain water content was significantly increased in the I/R + Yoh + Dex group $(\mathrm{P}<0.05)$ (see Figure 1 for further details).

\section{Cerebral infarction area measurement}

Cerebral infarction area measurements were performed on TTC-stained brain slices $24 \mathrm{~h}$ after ischemia. The results showed that no infarct was found in the Sham, Sham + Dex, Sham + Yoh + Dex, or Sham + SP600125 groups $(\mathrm{P}>0.05)$. Compared with the Sham group, the rats in the I/R, I/R + Dex, I/R + Yoh + Dex, and I/R + SP600125 groups had more cerebral infarction area $(\mathrm{P}<0.05)$, and compared with the I/R group, the cerebral infarction area in I/R + Dex, I/R + Yoh + Dex, and I/R + SP600125 groups was significantly decreased $(\mathrm{P}<0.05)$. The cerebral infarction area in the $I / R$ + Yoh + Dex group were significantly higher than those in the $\mathrm{I} / \mathrm{R}+$ Dex group $(\mathrm{P}<0.05)$ (see Figure 2$)$.

\section{fNK, and cleaved caspase-3 levels were determined by IF}

\section{Expression of p-JNK}

A small number of p-JNK-positive cells were observed in the Sham, Sham + Dex, Sham + Yoh + Dex, and Sham + SP600125 groups $(\mathrm{P}>0.05)$. Compared with the Sham group, the p-JNK-positive cells in the I/R, I/R + Dex, I/R + Yoh + Dex, and I/R + SP600125 groups and the expression of the p-JNK were significantly increased, and $(\mathrm{P}<0.05)$. Compared with the I/R group, the expression of $\mathrm{p}-\mathrm{JNK}$ in 


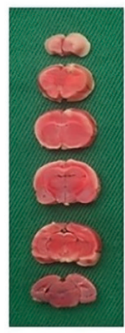

Sham

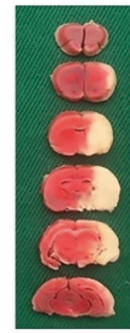

I/R

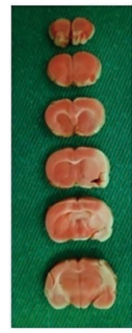

I/R + Dex I/R +Yoh + Dex I/R +SP600125

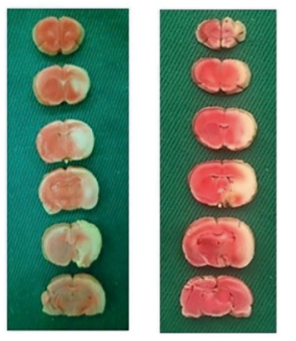

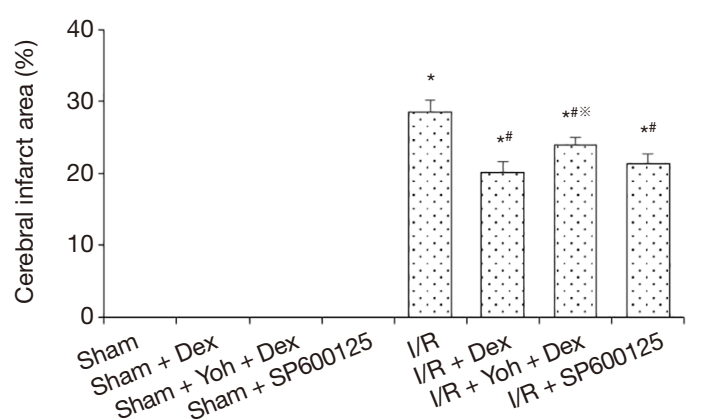

Figure 2 Cerebral infarction area in each group. (A) Cerebral infarct area stained with TTC. (B) Comparison of cerebral infarction area in each group $(\mathrm{n}=6) .{ }^{*}$ compared with the Sham group, $\mathrm{P}<0.05 ;{ }^{*}$ compared with the I/R group, $\mathrm{P}<0.05 ;{ }^{*}$ compared with the I/R $+\mathrm{Dex}$ group, $\mathrm{P}<0.05$. Dex, dexmedetomidine; Yoh, yohimbine; I/R, ischemic reperfusion; TTC, 2, 3, 5-triphenyltetrazolium chloride.
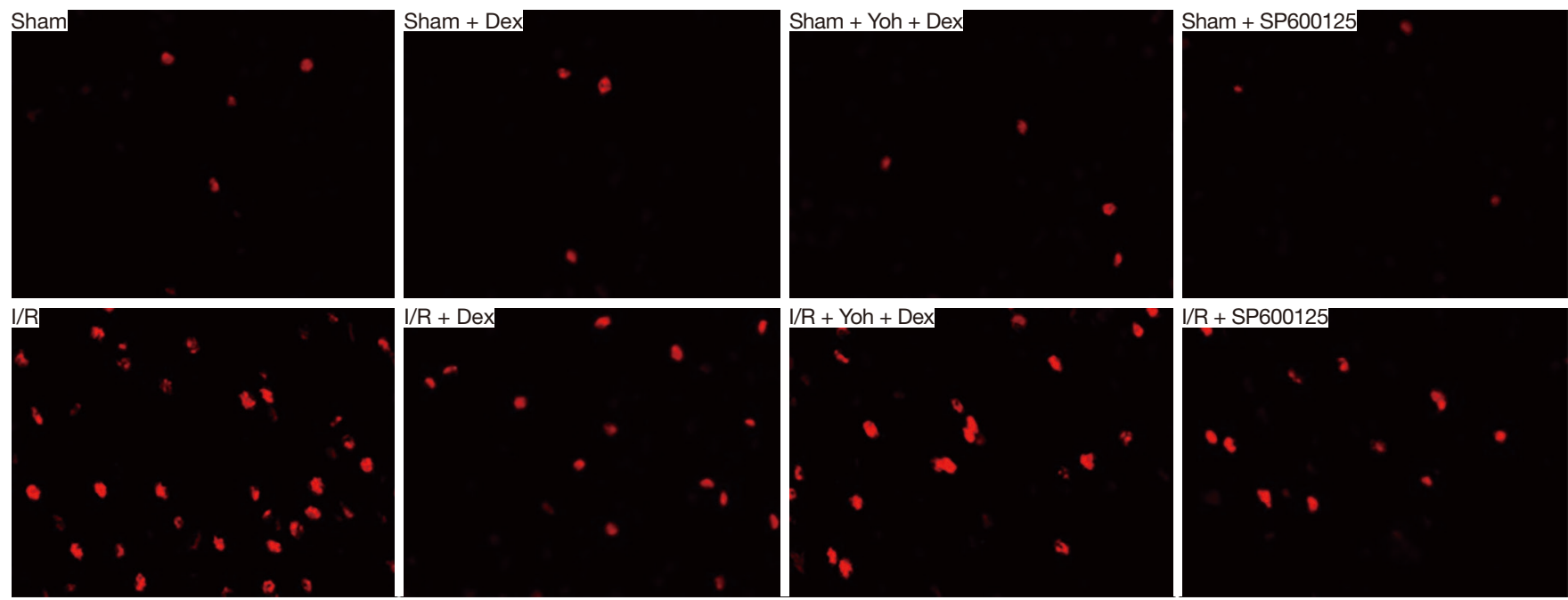

Figure 3 Immunofluorescence staining of p-JNK in each group. Magnification, 200x. Dex, dexmedetomidine; Yoh, yohimbine; I/R, ischemic reperfusion; JNK, Jun N-terminal kinase.

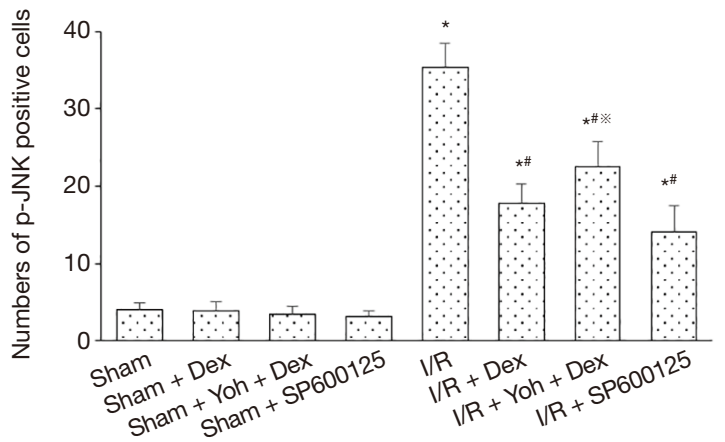

Figure 4 Comparison of $\mathrm{p}-\mathrm{JNK}$ positive cells in each group $(\mathrm{n}=6) .{ }^{*}$ compared with the Sham group, $\mathrm{P}<0.05$; ${ }^{*}$ compared with the I/R group, $\mathrm{P}<0.05$; * compared with the I/R + Dex group, $\mathrm{P}<0.05$. JNK, Jun N-terminal kinase; Dex, dexmedetomidine; Yoh, yohimbine; I/R, ischemic reperfusion.
$\mathrm{I} / \mathrm{R}+$ Dex, $\mathrm{I} / \mathrm{R}+$ Yoh + Dex, and I/R + SP600125 groups were significantly decreased $(\mathrm{P}<0.05)$, and the expression of the $\mathrm{p}-\mathrm{JNK}$ in the I/R + Yoh + Dex group were significantly increased compared with those in the $\mathrm{I} / \mathrm{R}+$ Dex group $(\mathrm{P}<0.05)$ (see Figures 3,4). Immunofluorescence double labeling revealed the co-localization of active caspase- 3 with GFAP, suggesting that there were astrocytes (Figure 5).

\section{Expression of cleaved caspase-3}

A small number of cleaved caspase- 3 positive cells were observed in the Sham, Sham + Dex, Sham + yohimbine (Yoh) + Dex, and Sham + SP600125 groups $(\mathrm{P}>0.05)$. Compared with the Sham group, the cleaved caspase- 3 positive cells 

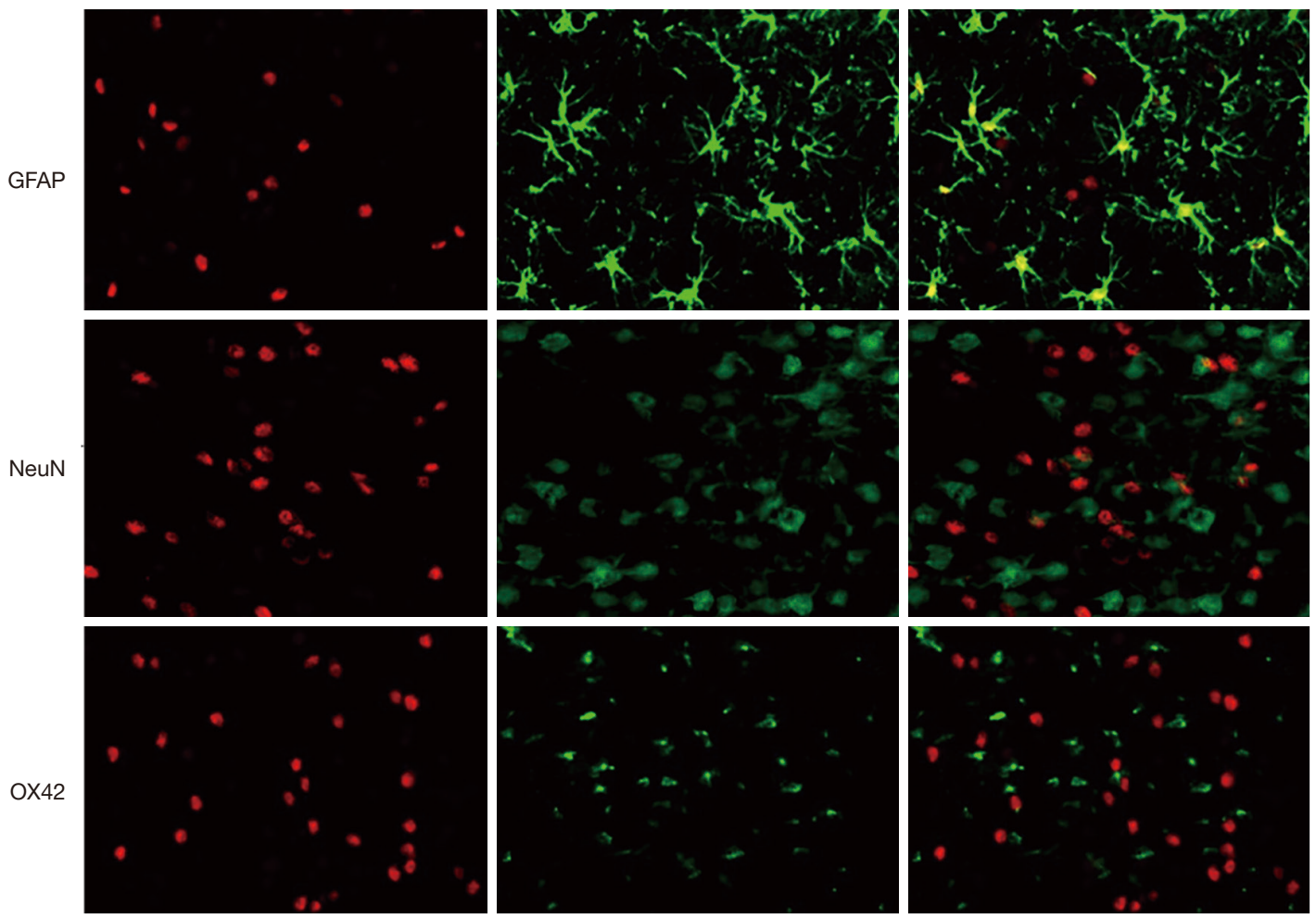

Figure 5 Double immunofluorescence staining with p-JNK in I/R group. Magnification, 200×. GFAP, Glial fibrillary acidic protein; JNK, Jun N-terminal kinase; I/R, ischemic reperfusion.

in $\mathrm{I} / \mathrm{R}, \mathrm{I} / \mathrm{R}+\mathrm{Dex}, \mathrm{I} / \mathrm{R}+$ Yoh + Dex, and I/R + SP600125 groups and the expression of $\mathrm{p}-\mathrm{JNK}$ were significantly increased $(\mathrm{P}<0.05)$, and compared with the $I / R$ group, the expression of the cleaved caspase- 3 in $\mathrm{I} / \mathrm{R}+\mathrm{Dex}, \mathrm{I} / \mathrm{R}+$ Yoh + Dex, and I/R + SP600125 groups were significantly decreased $(\mathrm{P}<0.05)$. The expression of the cleaved caspase- 3 in the $\mathrm{I} / \mathrm{R}+$ Yoh + Dex group were significantly increased compared with those in the $\mathrm{I} / \mathrm{R}+$ Dex group $(\mathrm{P}<0.05)$ (see Figures 6,7).

\section{The expression of $7 N K$ and $p-\mathcal{F N K}$ were determined by $W B$}

The expression of JNK showed no significance in the Sham, Sham + Dex, Sham + yohimbine (Yoh) + Dex, and Sham + SP600125 groups $(\mathrm{P}>0.05)$. Compared with the Sham group, the expression of p-JNK in I/R, I/R + Dex, I/R + Yoh + Dex, and I/R + SP600125 groups was significantly increased $(\mathrm{P}<0.05)$, and compared with the $\mathrm{I} / \mathrm{R}$ group, the expression of p-JNK in I/R + Dex, I/R + Yoh + Dex, and I/ $\mathrm{R}+\mathrm{SP} 600125$ groups was significantly decreased $(\mathrm{P}<0.05)$. The expression of $\mathrm{p}-\mathrm{JNK}$ in the I/R + Yoh + Dex group was significantly increased compared with the $\mathrm{I} / \mathrm{R}+$ Dex group $(\mathrm{P}<0.05)$ (see Figures 8,9).

\section{Discussion}

$\mathrm{CI} / \mathrm{RI}$ is a complex pathological process including oxidative stress, inflammation, calcium overload, mitochondrial dysfunctions, and excitotoxicity synergistically, which finally leads to dysfunction. In this study, neuronal death in the cortex, hippocampus, and striatum was induced by necrosis and apoptosis. The form of cell injury was determined by ischemia, which can cause many ischemic cells to die from necrosis or apoptosis in the ischemic core area. However, cells located in the ischemic penumbra induced apoptosis with short hypoxia time and a mild degree of ischemia. The primary goal for CI/RI therapy is to salvage tissue in the ischemic penumbra, and to develop drugs designed to inhibit apoptosis.

Protein kinases of the MAPK family can phosphorylate apoptotic proteins or anti-apoptotic proteins, and cause potential changes or signal transduction, which affects cell differentiation and apoptosis. The MAPK family comprises 

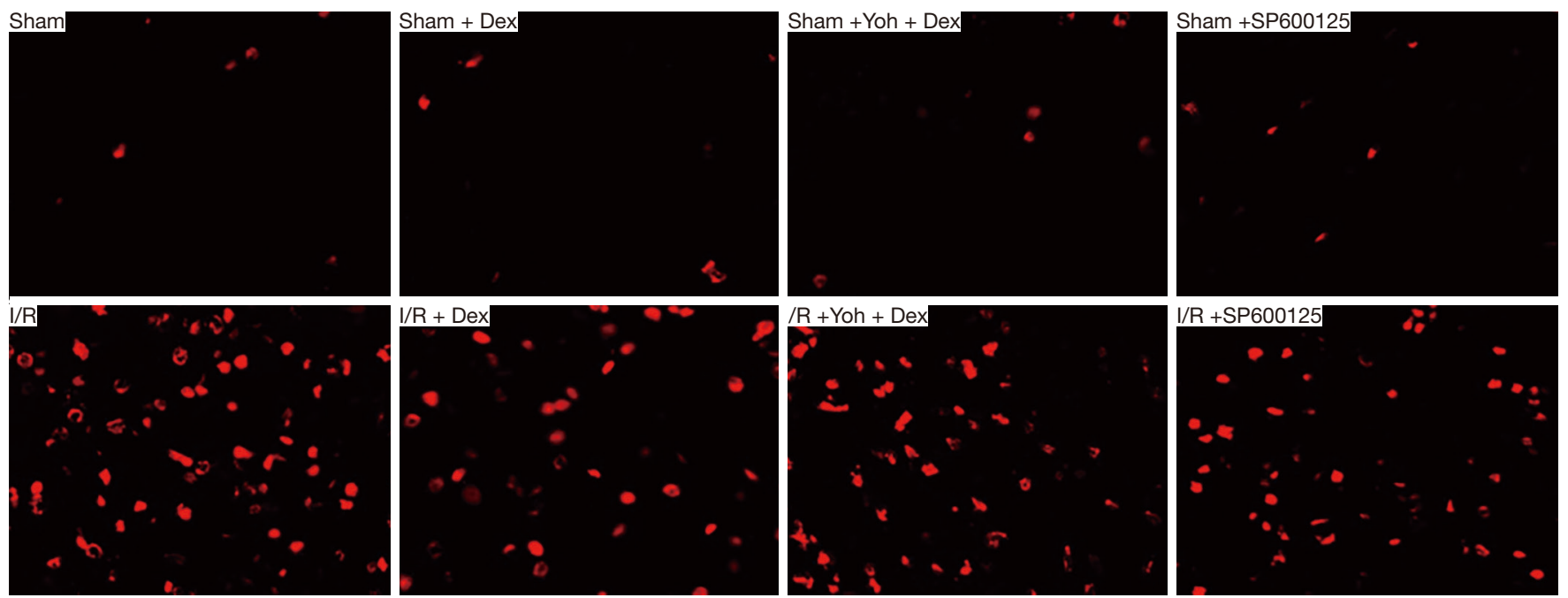

Figure 6 Immunofluorescence staining with cleaved caspase-3. Magnification, 200x. Dex, dexmedetomidine; Yoh, yohimbine; I/R, ischemic reperfusion.

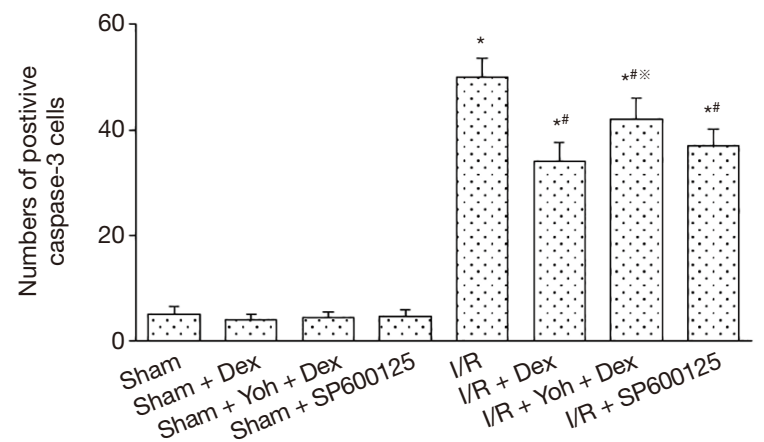

Figure 7 Comparison of cleaved caspase- 3 positive cells in each group ( $\mathrm{n}=6)$. * compared with the Sham group, $\mathrm{P}<0.05 ;{ }^{*}$ compared with the I/R group, $\mathrm{P}<0.05$; * compared with the I/R + Dex group, $\mathrm{P}<0.05$. Dex, dexmedetomidine; Yoh, yohimbine; I/R, ischemic reperfusion.

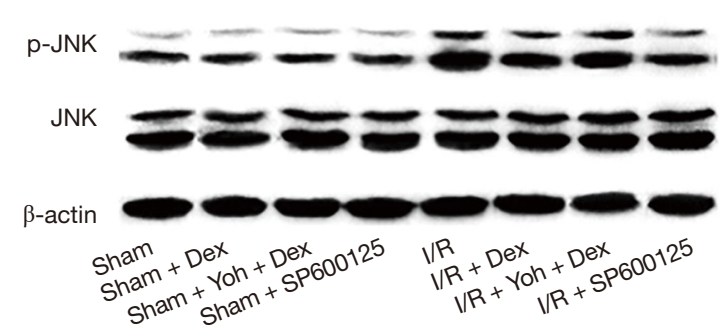

Figure 8 Protein expression levels of JNK and p-JNK were determined by western blotting in each group. JNK, Jun N-terminal kinase; Dex, dexmedetomidine; Yoh, yohimbine; I/R, ischemic reperfusion.

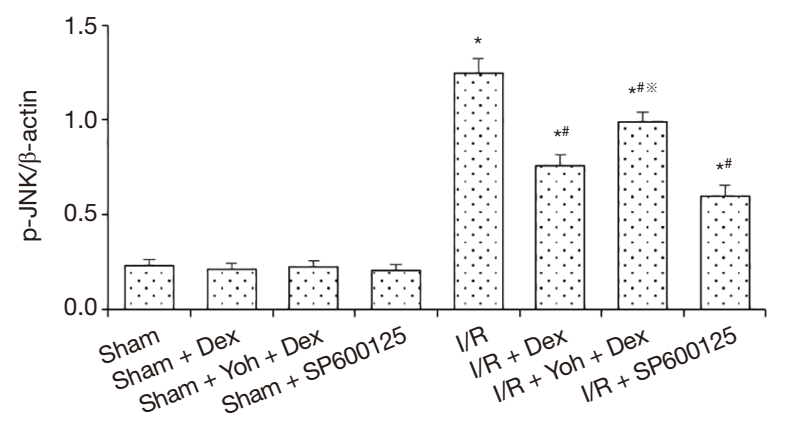

Figure 9 Comparison of p-JNK in each group $(n=6) .{ }^{*}$ compared with the Sham group, $\mathrm{P}<0.05$; \# compared with the I/R group, $\mathrm{P}<0.05 ;{ }^{*}$ compared with the I/R + Dex group, $\mathrm{P}<0.05$. JNK, Jun $\mathrm{N}$-terminal kinase; Dex, dexmedetomidine; Yoh, yohimbine; I/R, ischemic reperfusion.

three main parallel pathways: ERK, JNK, and p38, which are activated in CI/RI leading to a series of biological changes. The ERK pathway regulates cell proliferation, after which phosphorylation prevents cell apoptosis, and the p38 and JNK pathways promote cell apoptosis and speed up the death rate of brain cells in the ischemic area. Various extracellular stimuli activate JNK through a series of cascaded reactions, and change the balance of apoptotic anti-apoptotic proteins through endogenous and exogenous apoptotic pathways, finally leading to apoptosis. Caspase-3 plays a central role in cerebral ischemia injury and apoptosis, 
and both endogenous and exogenous apoptosis are finally realized through its activation. Activated caspase- 3 can also block DNA repair by cleaving the nuclear DNA repair enzyme PARP, leading to cell apoptosis.

SP600125, known as anthrapyrazolone, is a small molecule and highly selective inhibitor of the JNK pathway which is insoluble in water but not in DMSO (8). In one study SP600125 reversibly competed with ATP for binding sites and significantly inhibited JNK and c-Jun phosphorylation to block signal transduction (9). Lateral ventricular injection of blocker has a definite effect and small dosage, and in most studies, lateral ventricle injection of $10 \mathrm{iL}$ is used $30 \mathrm{~min}$ before ischemia (10).

Dex is a novel and highly selective á2 adrenergic receptor agonist that can produce sympathetic inhibition, sedative hypnotism, analgesia, reduction of general anesthesia dosage and side effects, and its sedative characteristics of easy arousal and no respiratory depression make it widely used in surgery and ICU sedation. By binding to á2 adrenergic receptors, Dex has a variety of physiological functions which are widely distributed in the central nervous system, peripheral nervous system, and autonomic ganglia. á2 adrenergic receptors are transmembrane G-protein coupled receptors with highest densities in the pons, parahippocampal gyrus, and locus ceruleus, where the agonist binds to the receptor, and the receptor protein changes conformation and activates the $G$ protein. The activated $G$ protein acts in the following ways: (I) phosphatidylinositol-specific phospholipase $\mathrm{C}$ is activated by $\mathrm{G}_{0}$ protein; (II) inhibition of $\mathrm{Ca}^{2+}$ transport by $\mathrm{G}_{0}$ protein; (III) inhibition of adenylate cyclase by $\mathrm{G}_{\mathrm{i}}$ protein; (IV) open the outward $\mathrm{K}^{+}$channel through $\mathrm{G}_{\mathrm{i}}$ protein; (V) through an unknown $G$ protein; (G) Adjust the exchange of $\mathrm{H}^{+}$and $\mathrm{Na}^{+}(11)$.

The protective effect of Dex on cerebral ischemia reperfusion injury was first reported by Hoffman (12). An experiment using a right common carotid artery insufficiency blocking I/R model was established with a low blood pressure method to reduce the concentration of plasma epinephrine and norepinephrine and minimize the caudate nucleus and hippocampal infarction area. After using the á adrenergic receptor Atipamezole, the protective effect of Dex decreased significantly, which showed that Dex could alleviate nerve injury by activating á 2 receptors and reducing the release of adrenaline and norepinephrine. With further research, the exact mechanism of Dex on nerve protection gradually became clear, revealing several aspects. Firstly, by reducing excitatory neurotransmitter release, the binding of Dex and $a_{2 \mathrm{~A}}$ can activate glutaminase, boosting the glutamine metabolism in astrocytes to reduce its toxic effects (13). Secondly, by inhibiting the release of catecholamine, Dex can inhibit central sympathetic nervous system activity and significantly reduce the level of circulating catecholamine, thus stabilize the balance of oxygen supply and demand in the brain and playing a neuroprotective role (14). Thirdly, Dex has a role in the inhibition of apoptosis and nutrition of nerves. In vitro experiments have shown that in hippocampal sections cultured with ischemic glucose deficiency, Dex pretreatment increases FAK phosphorylation and decreases Caspase-3 expression to improve cell survival (9). Dex can activate the PI3K/Akt and ERK1/2 signaling pathways and inhibit the phosphorylation of downstream GSK-3â to reduce the size of cerebral infarction area and the degree of edema, and improve neuronal survival (11). Dex can also up-regulate the expression of anti-apoptotic proteins Bcl-2 and MDM-2 and down-regulate the expression of pro-apoptotic protein Bax to reduce neuronal apoptosis (15). Fourthly, Dex plays a role in inhibiting inflammatory responses. In the study of Peng et al. (16), Dex reduced LPS induced neuronal injury in a dose-dependent manner by blocking the production of IL-â and TNF-á in microglia cells and inhibiting the inflammatory response. Wang et al. (17) found that Dex could reduce the contents of malondialdehyde, TNF-á, and IL-â in the brain tissues of rats with cerebral ischemia reperfusion injury to improve SOD activity and exert antiinflammatory and antioxidant effects.

Our results showed that compared with the I/R group, the rats in the Dex treatment group had a better mental state, lower neurological function scores, less defect symptoms, and a reduced cerebral infarct area and edema after $90 \mathrm{~min}$ of ischemia and $24 \mathrm{~h}$ of reperfusion. Many studies have demonstrated that Dex can ameliorate the histological changes and neurological dysfunction caused by I/R injury. Wang et al. (18) found that Dex could significantly improve nerve damage and brain tissue edema caused by subarachnoid hemorrhage and reduced the permeability of the blood-brain barrier. Clinical trials have also confirmed that Dex can improve postoperative cognitive dysfunction caused by clamping of the common artery during carotid artery excision (19).

After treatment with Dex, the number of p-JNK and cleaved caspase-3 positive cells, as well as p-JNK expression, were significantly lower than those in the I/R group, suggesting that Dex can inhibit the phosphorylation of JNK and reduce the activation of caspase-3. In 
Lipopolysaccharide (LPS)-induced acute lung injury models, Dex alleviated inflammatory cell infiltration and pulmonary edema by inhibiting JNK phosphorylation (20). In addition, Dex could improve postoperative cognitive function by reducing the expression of Caspase- 3 in rat brain tissue and inhibiting nerve cell apoptosis (21).

Most current studies conclude that the protective effect of Dex on I/R is mainly achieved through the activation of á2 adrenergic receptors which was also confirmed in our experiment (22). Compared with the $\mathrm{I} / \mathrm{R}+$ Dex group, rats in the $\mathrm{I} / \mathrm{R}+$ Yoh + Dex group had more severe neurological defect symptoms, higher scores, larger infarcted areas, and higher degrees of edema, and the number and expression of p-JNK and cleaved caspase-3 positive cells also increased. These results indicated Yoh could antagonize the inhibitory effect of Dex on JNK activation and weaken its protective effect against I/R. Therefore, we can infer that the cerebral protective effect of Dex may be related to the activation of á2 adrenergic receptors.

In summary, the results of this study showed that increased JNK phosphorylation and caspase-3 activation occur in CI/RI. Further, inhibition of JNK phosphorylation could reduce the activation of caspase- 3 in apoptotic practitioners and alleviate ischemia reperfusion injury, and the possible mechanism of Dex alleviating cerebral I/R injury was the activation of á2 adrenergic receptors, downregulation of JNK phosphorylation, and inhibition of caspase- 3 activation.

Due to the limited time and funding of the experiment, this study only referred to previous literature and selected a single dose of Dex without setting the concentration gradient to explore its optimal dose for neuroprotective effect. In addition, there were no multiple time points to observe the changes of various experimental indicators, which will be investigated in further experiments.

\section{Acknowledgments}

Funding: None.

\section{Footnote}

Reporting Checklist: The authors have completed the ARRIVE reporting checklist. Available at https://dx.doi. org/10.21037/apm-21-1218

Data Sharing Statement: Available at https://dx.doi. org/10.21037/apm-21-1218
Conflicts of Interest: All authors have completed the ICMJE uniform disclosure form (available at https://dx.doi. org/10.21037/apm-21-1218). The authors have no conflicts of interest to declare.

Ethical Statement: The authors are accountable for all aspects of the work in ensuring that questions related to the accuracy or integrity of any part of the work are appropriately investigated and resolved. Experiments were performed under a project license [NO.: SCXK (Yu) 20150004] granted by the ethics committee of the First Affiliated Hospital of Zhengzhou University, in compliance with Chinese national guidelines for the care and use of animals.

Open Access Statement: This is an Open Access article distributed in accordance with the Creative Commons Attribution-NonCommercial-NoDerivs 4.0 International License (CC BY-NC-ND 4.0), which permits the noncommercial replication and distribution of the article with the strict proviso that no changes or edits are made and the original work is properly cited (including links to both the formal publication through the relevant DOI and the license). See: https://creativecommons.org/licenses/by-nc$\mathrm{nd} / 4.0 /$.

\section{References}

1. Di Y, Lei $Y, Y u$ F, et al. MicroRNAs expression and function in cerebral ischemia reperfusion injury. J Mol Neurosci 2014;53:242-50.

2. Li C, Wang T, Zhang C, et al. Quercetin attenuates cardiomyocyte apoptosis via inhibition of JNK and p38 mitogen-activated protein kinase signaling pathways. Gene 2016;577:275-80.

3. Dong X, Xing Q, Li Y, et al. Dexmedetomidine protects against ischemia-reperfusion injury in rat skeletal muscle. J Surg Res 2014;186:240-5.

4. Schoeler M, Loetscher PD, Rossaint R, et al. Dexmedetomidine is neuroprotective in an in vitro model for traumatic brain injury. BMC Neurol 2012;12:20.

5. Cui J, Zhao H, Yi B, et al. Dexmedetomidine Attenuates Bilirubin-Induced Lung Alveolar Epithelial Cell Death In Vitro and In Vivo. Crit Care Med 2015;43:e356-68.

6. Liao Z, Cao D, Han X, et al. Both JNK and P38 MAPK pathways participate in the protection by dexmedetomidine against isoflurane-induced neuroapoptosis in the hippocampus of neonatal rats. Brain Res Bull 2014;107:69-78. 
7. Longa EZ, Weinstein PR, Carlson S, et al. Reversible middle cerebral artery occlusion without craniectomy in rats. Stroke 1989;20:84-91.

8. Urrutia A, Rubio-Araiz A, Gutierrez-Lopez MD, et al. A study on the effect of JNK inhibitor, SP600125, on the disruption of blood-brain barrier induced by methamphetamine. Neurobiol Dis 2013;50:49-58.

9. Jung EJ, Park HC, Chung KH, et al. Proteomic analysis of SP600125-controlled TrkA-dependent targets in SK-

N-MC neuroblastoma cells: inhibition of TrkA activity by SP600125. Proteomics 2014;14:202-15.

10. Ramin M, Azizi P, Motamedi F, et al. Inhibition of JNK phosphorylation reverses memory deficit induced by $\beta$-amyloid (1-42) associated with decrease of apoptotic factors. Behav Brain Res 2011;217:424-31.

11. Gerresheim G, Schwemmer U. Dexmedetomidine. Anaesthesist 2013;62:661-74.

12. Hoffman WE, Kochs E, Werner C, et al. Dexmedetomidine improves neurologic outcome from incomplete ischemia in the rat. Reversal by the alpha 2-adrenergic antagonist atipamezole. Anesthesiology 1991;75:328-32.

13. Huang R, Chen Y, Yu AC, et al. Dexmedetomidineinduced stimulation of glutamine oxidation in astrocytes: a possible mechanism for its neuroprotective activity. J Cereb Blood Flow Metab 2000;20:895-8.

14. Ma D, Rajakumaraswamy N, Maze M. alpha2Adrenoceptor agonists: shedding light on neuroprotection? Br Med Bull 2004;71:77-92.

15. Bell MT, Puskas F, Bennett DT, et al. Dexmedetomidine, an $\alpha$-2a adrenergic agonist, promotes ischemic tolerance

Cite this article as: Zhao B, Li D, Zhang $\mathrm{S}$, He L, Ai Y. Dexmedetomidine attenuates cerebral ischemia-reperfusion injury in rats by inhibiting the JNK pathway. Ann Palliat Med 2021;10(6):6768-6778. doi: 10.21037/apm-21-1218 in a murine model of spinal cord ischemia-reperfusion. J Thorac Cardiovasc Surg 2014;147:500-6.

16. Peng M, Wang YL, Wang CY, et al. Dexmedetomidine attenuates lipopolysaccharide-induced proinflammatory response in primary microglia. J Surg Res

2013;179:e219-25.

17. Wang Z, Kou D, Li Z, et al. Effects of propofoldexmedetomidine combination on ischemia reperfusioninduced cerebral injury. NeuroRehabilitation 2014;35:825-34.

18. Wang Y, Han R, Zuo Z. Dexmedetomidine post-treatment induces neuroprotection via activation of extracellular signal-regulated kinase in rats with subarachnoid haemorrhage. Br J Anaesth 2016;116:384-92.

19. Ge YL, Li X, Gao JU, et al. Beneficial effects of intravenous dexmedetomidine on cognitive function and cerebral injury following a carotid endarterectomy. Exp Ther Med 2016;11:1128-34.

20. Xu Y, Zhang R, Li C, et al. Dexmedetomidine attenuates acute lung injury induced by lipopolysaccharide in mouse through inhibition of MAPK pathway. Fundam Clin Pharmacol 2015;29:462-71.

21. Qian XL, Zhang W, Liu MZ, et al. Dexmedetomidine improves early postoperative cognitive dysfunction in aged mice. Eur J Pharmacol 2015;746:206-12.

22. Zhang XY, Liu ZM, Wen SH, et al. Dexmedetomidine administration before, but not after, ischemia attenuates intestinal injury induced by intestinal ischemia-reperfusion in rats. Anesthesiology 2012;116:1035-46.

(English Language Editor: B. Draper) 